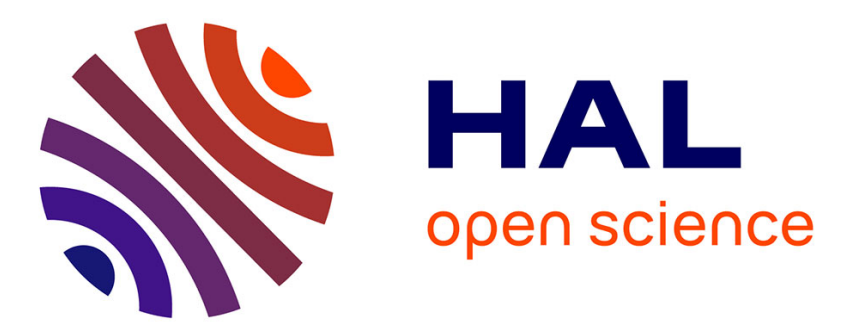

\title{
Adaptive Observer for Motorcycle State Estimation and Tire Cornering Stiffness Identification
}

\author{
Majda Fouka, Lamri Nehaoua, Mohammed El Habib Dabladji, Hichem \\ Arioui, Saïd Mammar
}

\section{- To cite this version:}

Majda Fouka, Lamri Nehaoua, Mohammed El Habib Dabladji, Hichem Arioui, Saïd Mammar. Adaptive Observer for Motorcycle State Estimation and Tire Cornering Stiffness Identification. 57th IEEE Conference on Decision and Control (CDC 2018 ), Dec 2018, Miami Beach, FL, United States. pp.30183024, 10.1109/CDC.2018.8619637 . hal-02063555

\section{HAL Id: hal-02063555 \\ https://hal.science/hal-02063555}

Submitted on 19 Dec 2019

HAL is a multi-disciplinary open access archive for the deposit and dissemination of scientific research documents, whether they are published or not. The documents may come from teaching and research institutions in France or abroad, or from public or private research centers.
L'archive ouverte pluridisciplinaire HAL, est destinée au dépôt et à la diffusion de documents scientifiques de niveau recherche, publiés ou non, émanant des établissements d'enseignement et de recherche français ou étrangers, des laboratoires publics ou privés. 


\title{
Adaptive Observer for Motorcycle State Estimation and Tire Cornering Stiffness Identification
}

\author{
M. Fouka, L. Nehaoua, M-H. Dabladji, H. Arioui and S. Mammar
}

\begin{abstract}
In this paper, a linear parameter varying (LPV) adaptive observer is designed for state estimation and tire cornering stiffness identification based on lateral motorcycle model. The estimation is based on a general Lipstchitz condition, Lyapunov function and is subjected to persistency of excitation conditions. Further, the LPV observer is transformed into Takagi-Sugeno (T-S) fuzzy observer and sufficient conditions, for the existence of the estimator, are given in terms of linear matrix inequalities (LMIs). This method is designed assuming that some of the states are not available, since parametric identification is generally developed assuming that all the system states are available (measured or estimated). Finally, the effectiveness of the proposed estimation method is illustrated through test scenarios performed with the wellknown motorcycle simulator "BikeSim" and by field test using data measurement carried out on experimental motorcycle.

Index Terms-Motorcycle, TS model, Luenberger Observer, Estimation, LMI.
\end{abstract}

\section{INTRODUCTION}

The technological progress of the powered two wheeled vehicles (PTWv) and its proliferation not only brought a noteworthy convenience to its users through a significant reduction of transportation time, but has given rise to serious safety issues as well. In the recent years, important researches [1] or [2] have been undertaken to investigate safer driving conditions in both normal and in critical situations, in order to improve security through the development of preventive and active safety systems. Complex riding assistance systems (ADAS) rely heavily on accurate and precise tools such as sensors that should be implemented on the motorcycle. While certain state variables, such as yaw rate, are measured directly, others, such as the tire cornering forces, cannot be measured due to high costs of some sensors leading inevitably to expensive new two wheelers. The ADAS systems are based on the lateral friction which its computing involves all the dynamic states of the motorcycle and a good modeling of the tire road contact. The use of model-based state estimators are therefore necessary to overcome previous shortcomings in order to provide estimates of unmeasured states and relevant parameters to achieve safety and handling.

The lateral slip conditions and the tire's adhesion limit are important for motorcycle stability in a turn, the capacity to maintain these conditions avoids dangerous side sliding leading to possible falls. The lateral tire forces are necessary

This work was supported by ANR project : VIROLO++ . All the authors are with the Informatique, Biologie Intgrative et Systmes Complexes Laboratory (IBISC), Evry Val d'Essonne University (UEVE), 40, rue de Pelvoux, 91020 Evry Courcouronne Cedex majda. fouka@univ-evry. fr to hold motorcycle through a turn, they are generated by the lateral tire deformation in the road contact. The major limitation of motorcycle dynamics is due to nonlinear tire characteristics and to loss of friction between tire and road. The lateral friction mainly depends on road condition (dry, wet, snow, ice) and is related to the efforts at the tire level (cornering stiffness). The estimation of motorcycle wheel interaction forces with the ground are very important, because of their influence on the stability of the two wheeled vehicle. This study focuses especially on the state and tire cornering coefficient estimation.

Considerable attention has been given to the development of safety systems over the past few years. Authors have investigated and developed different methods and different strategies to estimate motorcycle dynamic states for enhancing two wheelers stability and handling ([3]-[11]). Concerning lateral estimation most of cited papers consider restrictive assumptions which regarding riding motorcycle practices or constant longitudinal speed.

To the best knowledge of the author, there is only a few works about the estimation of the tires cornering parameters of the PTWv ([12] [13] [14]) Main researches are based on regression methods to estimate the current road conditions or the tire road contact.

This paper aims to estimate both out of plane motorcycle motion and the tire cornering stiffness coefficient by using a tire model. The tire model describes the relationship between the tire force and the slip as a function of two parameters, namely, tire cornering stiffness and tire camber stiffness. This estimation method based on the two bodies motorcycle model and a certain number of valid measurements in order to estimate in accurate way the tire coefficient of lateral force and the state vector. We assume a prior knowledge or classification of the road surface, the tires dynamic behavior is taken into account when evaluating the generated lateral tire forces.

In order to show the effectiveness of the estimation method, a tests were carried out on an instrumented motorcycle in realistic riding situations.

The rest of the paper is organized as follows. Sec. II, describes and discusses the motorcycle out of plane model and the tire behavior. Sec. III, illustrates the observer design and presents the convergence analysis. In Sec. IV, discuss the results, compared to real experimental data and evaluate the estimated model. Finally Sec. V, we make some concluding remarks regarding our study and future perspectives. 


\section{LATERAl DYNAMics of PTWV (OUT-OF-PLANE)}

In this paper, the out-of-plane dynamics of the motorcycle (figure 1) are modeled as proposed in Sharp's 1971 model [15] which consist of the lateral and steering ones [16]. The main frame is subject to lateral motion, roll motion and yaw motion and the front frame is subject to steering motion.

Assumption 1: Let us consider the following assumptions:

1) The rider is supposed rigidly attached to the main frame.

2) The forward velocity $v_{x}$ is the varying parameter and it is considered measurable,

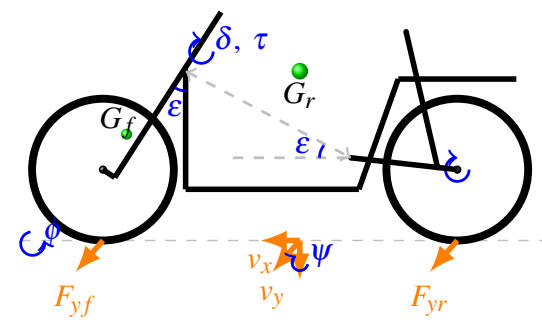

Fig. 1. Geometrical representation of the Sharp's motorcycle model

The motions of the motorcycle can be modeled by the following equations:

$$
\left\{\begin{array}{l}
e_{33} \dot{v}_{y}+e_{34} \ddot{\psi}+e_{35} \ddot{\phi}+e_{36} \ddot{\delta}=a_{34} \dot{\psi}+\sum F_{y} \\
e_{34} \dot{v}_{y}+e_{44} \ddot{\psi}+e_{45} \ddot{\phi}+e_{46} \ddot{\delta}=a_{44} \dot{\psi}+a_{45} \dot{\phi}+a_{46} \dot{\delta}+\sum M_{z} \\
e_{35} \dot{v}_{y}+e_{45} \ddot{\psi}+e_{55} \ddot{\phi}+e_{56} \ddot{\delta}=a_{54} \dot{\psi}+a_{56} \dot{\delta}+\sum M_{x} \\
e_{36} \dot{v}_{y}+e_{46} \ddot{\psi}+e_{56} \ddot{\phi}+e_{66} \ddot{\delta}=a_{64} \dot{\psi}+a_{65} \dot{\phi}+a_{66} \dot{\delta}+\sum M_{s} \\
\dot{F}_{y f}=a_{710} \phi+a_{720} \delta+a_{730} v_{y}+a_{740} \dot{\psi}+a_{760} \dot{\delta}+a_{770} F_{y f} \\
\dot{F}_{y r}=a_{810} \phi+a_{830} v_{y}+a_{840} \dot{\psi}+a_{880} F_{y r}
\end{array}\right.
$$

where:

$$
\left\{\begin{array}{l}
\sum F_{y}=F_{y f}+F_{y r}=M a_{y} \\
\sum M_{z}=a_{47} F_{y f}+a_{48} F_{y r} \\
\sum M_{x}=a_{51} \sin (\phi)+a_{52} \sin (\delta) \\
\sum M_{s}=a_{61} \sin (\phi)+a_{62} \sin (\delta)+a_{67} F_{y f}+\tau
\end{array}\right.
$$

Where $(\phi, \delta, \psi, \dot{\phi}, \dot{\delta}, \dot{\psi})$ denote the roll, steering, yaw angles and respectively their time derivatives, whereas $v_{y}$ is the lateral velocity, $F_{y f}$ and $F_{y r}$ are the cornering front and rear forces respectively, and $\tau$ is the torque applied to the handle bar. For further details on the motorcycle parameters $\left(e_{i j}, a_{i j}\right)$ and expressions refer to table $\mathrm{I}$.

\section{A. The Tyre Forces}

In this section a brief description of the tire/road contact forces are introduced to understand the tire dynamics and consequently the parameters of the tire model. The lateral tire forces are necessary to hold motorcycle through a turn, the major difficulties in motorcycle motion is the evaluation of the tire and road interaction [17]. The lateral front and rear forces $F_{y f}$ and $F_{y r}$, are generated when there is simultaneously side slip angles $\alpha$ and camber angles $\gamma$. The mathematical formulas of these lateral forces and angles, expressed in the motorcycle-related reference, are therefore given by :

$$
\left\{\begin{array}{l}
\frac{\sigma_{f}}{v_{x}} \dot{F}_{y f}=-F_{y f}+\Delta_{y f} \\
\frac{\sigma_{r}}{v_{x}} \dot{F}_{y r}=-F_{y r}+\Delta_{y r}
\end{array}\right.
$$

with

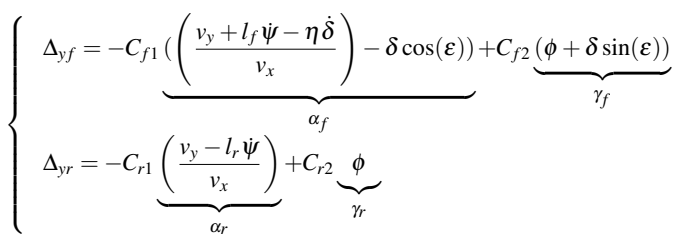

$C_{f i}$ and $C_{r i}$ refer to the tire forces coefficients (stiffness and camber coefficients $i=(1,2)), \varepsilon$ refers to the caster angle, $\eta$ is the mechanical trail, $l_{f}$ (resp. $l_{r}$ ) represents the distance between the center of mass and the front and rear axis. Considering the lateral forces in their linear form is not restrictive because we are targeting urban scenarios where the camber and slip angles remains in the linear domain of the lateral forces.

\section{B. Linearized Model Of Motorcycle}

From equations (1), we have a state representation with 8 states $x(t)=\left[\phi, \delta, v_{y}, \dot{\psi}, \dot{\phi}, \dot{\delta}, F_{y f}, F_{y r}\right]^{T}$. Sensors allow us to get the following variables:

$\bar{y}=\left(\delta, \dot{\psi}, \dot{\phi}, \dot{\delta}, a_{y}=\frac{F_{y f}+F_{y r}}{M}\right)$. Let us consider the following $\rho(t)=v_{x}(t)$ known. Thus, the Linear Parameter Varying (LPV) structure is expressed by:

$$
\left\{\begin{array}{c}
E \dot{x}(t)=A(\rho) x(t)+B \tau(t)+f\left(x, v_{x}, \theta\right) \\
\bar{y}=\bar{C} x(t)
\end{array}\right.
$$

where,

$$
\bar{y}=\underbrace{\left[\begin{array}{cccccccc}
0 & 1 & 0 & 0 & 0 & 0 & 0 & 0 \\
0 & 0 & 0 & 1 & 0 & 0 & 0 & 0 \\
0 & 0 & 0 & 0 & 1 & 0 & 0 & 0 \\
0 & 0 & 0 & 0 & 0 & 1 & 0 & 0 \\
0 & 0 & 0 & 0 & 0 & 0 & \frac{1}{M} & \frac{1}{M}
\end{array}\right]}_{\bar{C}} x(t)
$$

Whereas $x(t) \in \mathbb{R}^{n}$ and $y(t) \in \mathbb{R}^{n_{y}}$. The matrices $A \in \mathbb{R}^{n \times n}$, $B \in \mathbb{R}^{n \times 1}, \bar{C} \in \mathbb{R}^{n_{y} \times n} . f\left(x, v_{x}, \theta\right) \in \mathbb{R}^{n}$ depend essentially on the tire dynamics and it can be rewritten in the following linear form with respect to tire parameters:

$$
f\left(x, v_{x}, \theta\right)=D \chi\left(x, v_{x}\right) \theta
$$

Where,

$$
\chi=\left[\begin{array}{cccc}
-\frac{v_{x}}{\sigma_{f}} \alpha_{f} & \frac{v_{x}}{\sigma_{f}} \gamma_{f} & 0 & 0 \\
0 & 0 & -\frac{v_{x}}{\sigma_{r}} \alpha_{r} & \frac{v_{x}}{\sigma_{r}} \gamma_{r}
\end{array}\right], \theta=\left[\begin{array}{c}
C_{f 1}-C_{f 1} \\
C_{f 2}-C_{f 2} \\
C_{r 1}-C_{r 1} \\
C_{r 2}-C_{r 20}
\end{array}\right]=\left[\begin{array}{c}
\theta_{f 1} \\
\theta_{f 2} \\
\theta_{r 1} \\
\theta_{r 2}
\end{array}\right]
$$

Where, $C_{(f, r) i_{0}}$ are the prior value of the stiffness coefficient. The matrices $E, A, D$ and $B$ and a summary of variable nomenclature are given in the appendix. 


\section{Additional measurement}

The estimation approach requires to satisfy the observability condition of the pair $(A(\rho), \bar{C})$ for the system 4$)$ and the rank condition $\operatorname{rank}(\bar{C} D)=\operatorname{rank}(D)$ to estimate the unknown parameters (tire stiffness coefficient). In regards to the model (4) these conditions are not verified. We propose to use the flatness properties [19] in order to add virtual measures using differentiators [20].

From equations (1), one make some algebraic manipulations in order to obtain additional informations about lateral tire forces as follows:

$\left\{\begin{array}{c}M \dot{v}_{y}+M_{f} k \ddot{\psi}+\left(M_{f} j+M_{r} h\right) \ddot{\phi}+M_{f} e \ddot{\delta}=-M v_{x} \dot{\psi}+F_{y f}+F_{y r} \\ M_{f} k \dot{v}_{y}+e_{44} \ddot{\psi}+e_{45} \ddot{\phi}+e_{46} \ddot{\delta}=-M_{f} k v_{x} \dot{\psi}+a_{45} \dot{\phi}+\frac{i_{f y}}{R_{f}} \sin \varepsilon v_{x} \dot{\delta} \\ +l_{f} F_{y f}-l_{r} F_{y r}\end{array}\right.$

After slight calculations, one can write :

$$
\begin{array}{r}
\left(M_{f}^{2} k^{2}-M e_{44}\right) \ddot{\psi}+\left(M_{f} k\left(M_{f} j+M_{r} h\right)-M e_{45}\right) \ddot{\phi}+ \\
\left(M_{f} k M_{f} e-M e_{46}\right) \ddot{\delta}=-M a_{45} \dot{\phi}-M \frac{i_{f y}}{R_{f}} \sin \varepsilon v_{x} \dot{\delta}+ \\
\left(M_{f} k-M l_{f}\right) F_{y f}+\left(M_{f} k+M l_{r}\right) F_{y r}
\end{array}
$$

Hence, we can state that $\left(M_{f} k-M l_{f}\right) F_{y f}+\left(M_{f} k+M l_{r}\right) F_{y r}$ can be considered as an additional virtual measure thanks to the following algebraic estimator:

$$
\begin{array}{r}
\left(M_{f} k-M l_{f}\right) \hat{F}_{y f}+\left(M_{f} k+M l_{r}\right) \hat{F}_{y r}=\left(M_{f}^{2} k^{2}-M e_{44}\right) \hat{\dot{\psi}} \\
+\left(M_{f} k\left(M_{f} j+M_{r} h\right)-M e_{45}\right) \hat{\ddot{\phi}}+ \\
\left(M_{f} k M_{f} e-M e_{46}\right) \hat{\tilde{\delta}}+M a_{45} \hat{\dot{\phi}}+M \frac{i_{f y}}{R_{f}} \sin \varepsilon v_{x} \hat{\dot{\delta}}
\end{array}
$$

By considering the information in equation (8) as additional measure and $\left(M a_{y}=F_{y f}+F_{y r}\right)$, the new measurement vector becomes:

$$
y=\underbrace{\left[\begin{array}{c}
\bar{C} \\
C_{0}
\end{array}\right]}_{C} x
$$

with $C_{0}=\left[0,0,0,0,0,0,\left(M_{f} k-M l_{f}\right),\left(M_{f} k+M l_{r}\right)\right]$

The following study highlights the different steps used to obtain the TS model used in the design of the adaptive observer.

\section{EXACT T-S MOTORCYCLE MODEL}

In order to express the LPV model (4) in T-S fuzzy structure [18], let us take $v_{x}$ in the interval where the motorcycle is stable. Note that there is one nonlinearity that's why the system is described with 2 sub-models [6]. The variables $\zeta_{i}($.$) are the weighing functions and they must$ satisfy the following convex sum property:

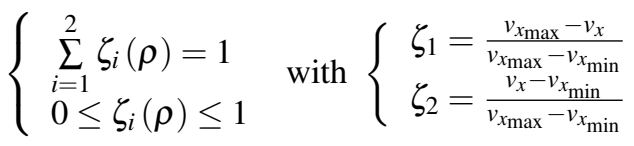

The model can be exactly expressed as follows:

$$
\left\{\begin{array}{ccc}
E \dot{x} & = & \sum_{i=1}^{2} \zeta_{i}(\rho) A_{i} x+B u(t)+f\left(x, v_{x}, \theta\right) \\
y & = & C x
\end{array}\right.
$$

Where, $A(\rho)=\sum_{i=1}^{2} \zeta_{i}(\rho) A_{i}$ and $E^{-1}$ exists.

\section{OBSERVER DESIGN}

In this section, we address the problem of state and parameters estimation for out of plane motorcycle dynamics by an adaptive observer.

Assumption 2: Throughout the paper, the following assumptions are considered:

- Suppose that the signals inputs $(u)$ is known and sufficiently persistent.

- The state vector $x(t)$ and the input are considered bounded (stable motorcycle case),

- The pair $\left(A_{i}, C\right)$ is observable $i=(1,2)$.

Let us denote $\hat{x}(t), \hat{y}(t)$ and $\hat{\theta}$ the estimated state, output vector and the parameters vector respectively. The structure of the observer in this case is :

$$
\left\{\begin{array}{ccc}
E \hat{\dot{x}} & = & A(\rho) \hat{x}+B u+f\left(\hat{x}, v_{x}, \hat{\theta}\right)+L(\rho)(y(t)-C \hat{x}) \\
\hat{y} & = & C \hat{x}
\end{array}\right.
$$

The matrix $L$ is parameter varying: $L(\rho)=\sum_{i=1}^{2} \zeta_{i}(\rho) L_{i}$. The state estimation error is given by: $\tilde{x}=x-\hat{x}$ and the parameters estimation error is $\tilde{\theta}=\theta-\hat{\theta}$.

\section{A. Observer's Convergence Study}

In this paper, we study the LPV luenberger observer design problem for the class of Lipschitz systems 4. where the function $f\left(x, v_{x}, \theta\right)$ satisfies the uniform global Lipschitz condition in $x$ [21]. Notice that any system of the form (4) can be expressed with the lemma (1) as long as $f\left(x, v_{x}, \theta\right)$ is continuously differentiable with respect to $x$.

Lemma 1: The continuous function $f\left(x, v_{x}, \theta\right)$ is said to be Lipschitz with respect to $x$, if for all $x, \hat{x} \in R^{n}$ it can be written in a generalized Lipschitz condition as:

$$
\tilde{f}^{T} Q \tilde{f} \leq \tilde{x}^{T} R \tilde{x}
$$

where $\tilde{f}=f\left(x, v_{x}, \theta\right)-\hat{f}\left(\hat{x}, v_{x}, \theta\right), \tilde{x}=x-\hat{x}, Q$ is a positive definite symmetric matrix and $R$ is a semi-positive definite symmetric matrices.

Please refer to the appendix for the key step to define the Lipschitz condition for the unknown part of the model $\tilde{f}$.

The stability analysis is performed by using a quadratic Lyapunov function as follows :

$$
V(\tilde{x})=\tilde{x}(t)^{T} P E \tilde{x}(t)+\tilde{\theta}^{T} \Gamma^{-1} \tilde{\theta}, \quad P=P^{T}>0
$$

The state estimation error obeys to the following differential equation:

$$
\begin{aligned}
& E \tilde{\dot{x}}(t)=A \tilde{x}+\left(f\left(x, v_{x}, \theta\right)-f\left(\hat{x}, v_{x}, \hat{\theta}\right)-L C(x-\hat{x})\right. \\
& E \tilde{\dot{x}}(t)=(A-L C) \tilde{x}+\left(f\left(x, v_{x}, \theta\right)-f\left(\hat{x}, v_{x}, \hat{\theta}\right)\right)+ \\
& \quad f\left(\hat{x}, v_{x}, \theta\right)-f\left(\hat{x}, v_{x}, \theta\right) \\
& E \tilde{\dot{x}}(t)=(A-L C) \tilde{x}+\tilde{f}\left(\tilde{x}, v_{x}, \theta\right)+D \chi\left(\hat{x}, v_{x}\right)(\theta-\hat{\theta}) \\
& E \tilde{\dot{x}}(t)=\Phi \tilde{x}+\tilde{f}+D \chi\left(\hat{x}, v_{x}\right) \tilde{\theta}
\end{aligned}
$$

With $\Phi=A-L C$.

The time derivative of the Lyapunov function is computed 
by using the state estimation error dynamics (14), as follow:

$$
\begin{aligned}
\dot{V} & =\tilde{x}^{T} E P^{T} \tilde{x}+\tilde{x}^{T} P E \tilde{x}+\tilde{\theta}^{T} \Gamma^{-1} \tilde{\dot{\theta}}+\tilde{\dot{\theta}}^{T} \Gamma^{-1} \tilde{\theta} \\
\dot{V} & =\left(\Phi \tilde{x}+\tilde{f}+D \chi\left(\hat{x}, v_{x}\right) \tilde{\theta}\right)^{T} P^{T} \tilde{x}+\tilde{x}^{T} P\left(\Phi \tilde{x}+\tilde{f}+D \chi\left(\hat{x}, v_{x}\right) \tilde{\theta}\right) \\
& +\tilde{\theta}^{T} \Gamma^{-1} \tilde{\dot{\theta}}+\tilde{\dot{\theta}}^{T} \Gamma^{-1} \tilde{\theta} \\
\dot{V} & =\tilde{x}^{T} \Phi^{T} P^{T} \tilde{x}+\tilde{f}^{T} P^{T} \tilde{x}+\tilde{x}^{T} P \Phi \tilde{x}+\tilde{x}^{T} P \tilde{f}+\tilde{\theta}^{T} \chi^{T}\left(\hat{x}, v_{x}\right) D^{T} P \tilde{x} \\
& +\tilde{\theta}^{T} \Gamma^{-1} \tilde{\dot{\theta}}+\tilde{\dot{\theta}}^{T} \Gamma^{-1} \tilde{\theta}+\tilde{x}^{T} P D \chi\left(\hat{x}, v_{x}\right) \tilde{\theta} \\
\dot{V} & =\tilde{x}^{T}\left(\Phi^{T} P^{T}+P \Phi\right) \tilde{x}+\tilde{f}^{T} P^{T} \tilde{x}+\tilde{x}^{T} P \tilde{f}+\tilde{\theta}^{T} \Gamma^{-1} \tilde{\dot{\theta}}+\tilde{\theta}^{T} \Gamma^{-1} \tilde{\theta}+ \\
& \tilde{\theta}^{T} \chi^{T}\left(\hat{x}, v_{x}\right) D^{T} P \tilde{x}+\tilde{x}^{T} P D \chi\left(\hat{x}, v_{x}\right) \tilde{\theta}
\end{aligned}
$$

Where $\Psi=\Phi^{T} P^{T}+P \Phi$.

Lemma 2: ([22]) For every matrix $G=G^{T}>0$, the property below is true:

$$
X^{T} Y+Y^{T} X \leq X^{T} G X+Y^{T} G^{-1} Y
$$

Using the Lipschitz condition (1) and lemma (2), one obtain the following inequality :

$$
\tilde{f}^{T} P^{T} \tilde{x}+\tilde{x}^{T} P \tilde{f} \leq \tilde{x}^{T} P Q^{-1} P^{T} \tilde{x}+\tilde{f}^{T} Q \tilde{f}
$$

Now, the derivative of Lyapunov function is as follows:

$$
\begin{gathered}
\dot{V}(t) \leq \tilde{x}^{T}\left(\Psi+P Q^{-1} P^{T}+R\right) \tilde{x}+\tilde{\theta}^{T} \chi^{T}\left(\hat{x}, v_{x}\right) D^{T} P \tilde{x}+ \\
\tilde{x}^{T} P D \chi\left(\hat{x}, v_{x}\right) \tilde{\theta}+\tilde{\theta}^{T} \Gamma^{-1} \tilde{\dot{\theta}}+\tilde{\dot{\theta}}^{T} \Gamma^{-1} \tilde{\theta}
\end{gathered}
$$

\section{B. The adaptive law :}

The adaptive law is defined from the derivative of the Lyapunov function in order to guarantee state asymptotic convergence. Thus, if the condition $\operatorname{rank}(C D)=\operatorname{rank}(D)$ is checked, we can find a matrix $\Gamma$ such that

$$
\left\{\begin{array}{l}
\tilde{\theta}^{T} \Gamma^{-1} \tilde{\dot{\theta}}=-\tilde{\theta}^{T} \chi^{T}\left(\hat{x}, v_{x}\right) D^{T} P \tilde{x} \\
\tilde{\dot{\theta}}^{T} \Gamma^{-1} \tilde{\theta}=-\tilde{x}^{T} P D \chi\left(\hat{x}, v_{x}\right) \tilde{\theta}
\end{array}\right.
$$

Note that $\dot{\tilde{\theta}}=-\dot{\hat{\theta}}(\dot{\theta}=0)$ and the two equations above are equivalent, it is possible to find a matrix $T$, such that $D^{T} P=$ $T C$ [25], then the parameters identification is done with the following equation:

$$
\begin{aligned}
\hat{\dot{\theta}}(t) & =\Gamma \chi^{T}\left(\hat{x}, v_{x}\right) T(y-\hat{y}) \\
& =\Gamma \chi^{T}\left(\hat{x}, v_{x}\right) T C(x-\hat{x}) \\
& =\Gamma \chi^{T}\left(\hat{x}, v_{x}\right) T C \tilde{x}
\end{aligned}
$$

Considering equality 15 , the time derivative $\dot{V}(t)$ is

$$
\dot{V}(t) \leq \tilde{x}^{T}\left(A^{T} P^{T}+P A-C^{T} K^{T}-K C+P Q^{-1} P^{T}+R\right) \tilde{x}
$$

Where $K=P L, \Phi=A-L C$ and $\Psi=\Phi^{T} P^{T}+P \Phi$.

Theorem 1: The state estimation error asymptotically converges toward zero if there exist a symmetric positive definite matrix $P \in \mathbb{R}^{n \times n}$ and a matrix $K \in \mathbb{R}^{n \times n y}$ such that the following linear matrix inequality holds

$$
P A(\rho)+A(\rho)^{T} P-K(\rho) C-C^{T} K(\rho)^{T}+P Q^{-1} P^{T}+R(\rho)<0
$$

From theorem 1 and using the convex sum property of the weighting functions, sufficient conditions ensuring $(\dot{V}(\tilde{x}(t))<0)$ are given by the following LMIs:

$$
\begin{aligned}
A_{i}^{T} P+P A_{i}-C^{T} K_{i}^{T}-K_{i} C+P Q^{-1} P^{T}+R_{i} & <0 \\
K_{i} & =P L_{i}
\end{aligned}
$$

This equation is a Linear Matrix Inequality (LMI) and it can be solved easily with Yalmip toolbox of Matlab to ensure the convergence of the observer.

In order to complete the proof, it remains to establish some LMI conditions to ensure that :

$$
\left\{\begin{array}{ccc}
D^{T} P & = & T C \\
P E & = & E P^{T}>0
\end{array}\right.
$$

We formulate the equality constraint $D^{T} P=T C$ as an optimization problem (introduced in [25]):

$$
\min \eta
$$

subject to

$$
\left[\begin{array}{cc}
\eta I & D^{T} P-T C \\
\left(D^{T} P-T C\right)^{T} & \eta I
\end{array}\right]>0
$$

From the LMI inequality (19) and 21, one compute the matrices $P, K_{i}, T$ and $L_{i}$. Then the observer given by the equations (12) will ensure the state estimation.

Moreover, if the Persistent Excitation Condition is checked, the unknown parameter convergence can also be obtained.

Definition 1: Persistence excitation [23]

The Persistent Excitation Condition is obtained if there exist $c_{1}, c_{2}$ and $c_{3}$ such that for all $t$ the following inequality holds:

$$
c_{1} I \leq \int_{t_{0}}^{t_{0}+c_{3}} D \chi\left(\hat{x}, v_{x}\right) \chi^{T}\left(\hat{x}, v_{x}\right) D^{T} d t \leq c_{2} I
$$

Remark 1: In practice, the excitation input is the rider torque, must be chosen in scenario as to well excited parameters to be identified.

\section{Simulation Results}

The simulations are carried out on a nonlinear model which takes account the lateral dynamics of PTWv. The rider's steering torque is the excitation input. The gains of the observer are computed from equations (19) and (21). The adaptation law provide an on-line estimation of the unknown stiffness parameters.

\section{A. Motorcycle BikeSim Simulation Test}

The proposed approach is evaluated under different driving scenarios using the well-known motorcycle's simulator: BikeSim based on the nonlinear multibody Sharp's 2004 model [24]. For a slalom maneuver with a velocity of $v_{x}=$ $50 \mathrm{~km} / \mathrm{h}$, steering torque input and the motorcycle path are depicted in fig. 2. The obtained results are given in figure 3 which show the estimated tire coefficient of the lateral model between nominal and estimated one, the state estimation for this scenarios are depicted in the figures 45
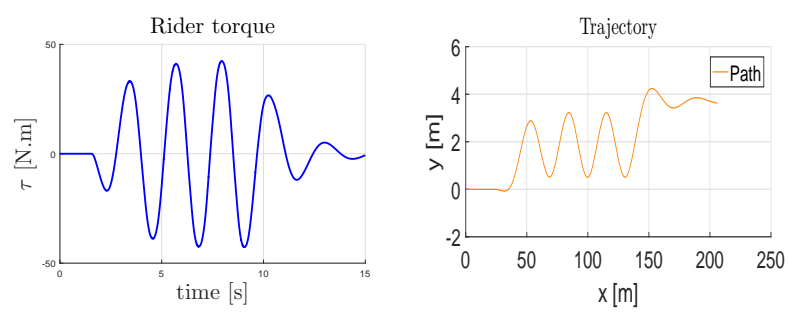

Fig. 2. (left) Rider torque $\tau$ (right) Longitudinal velocity - Path. 

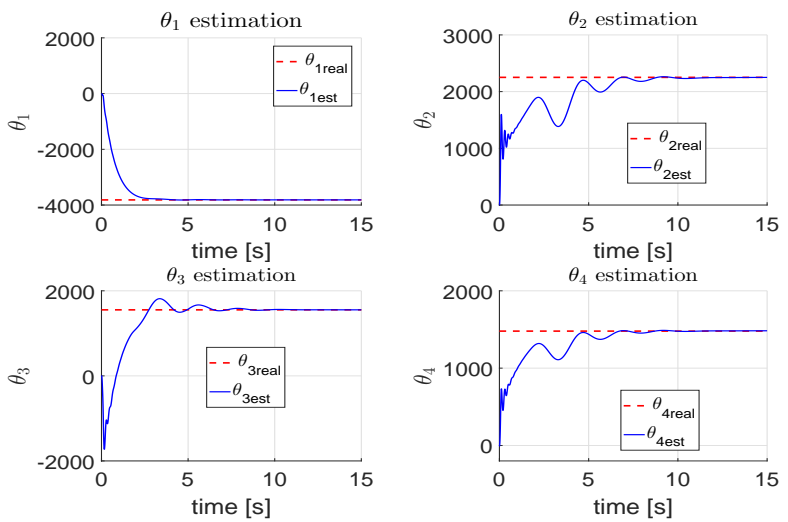

Fig. 3. Convergence of parameters estimates $\hat{\theta}_{1}, \hat{\theta}_{2}, \hat{\theta}_{3}$ and $\hat{\theta}_{4}$, real parameters value are shown by dashed.
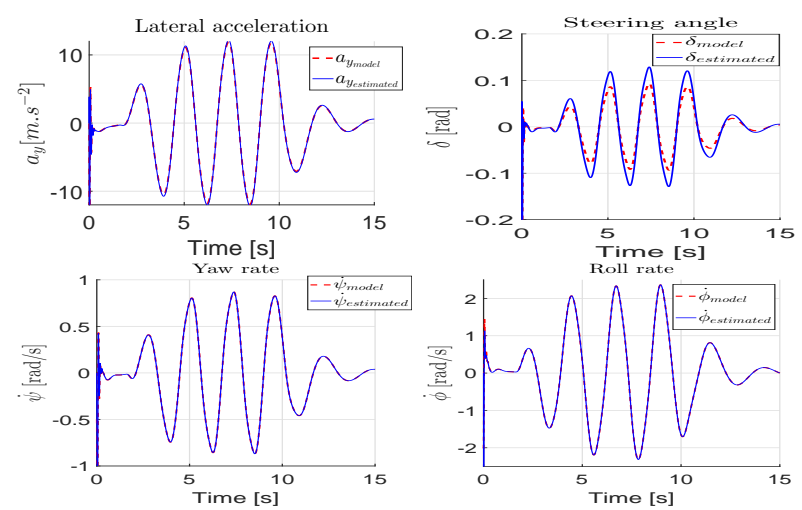

Fig. 4. States estimation compared to output state.
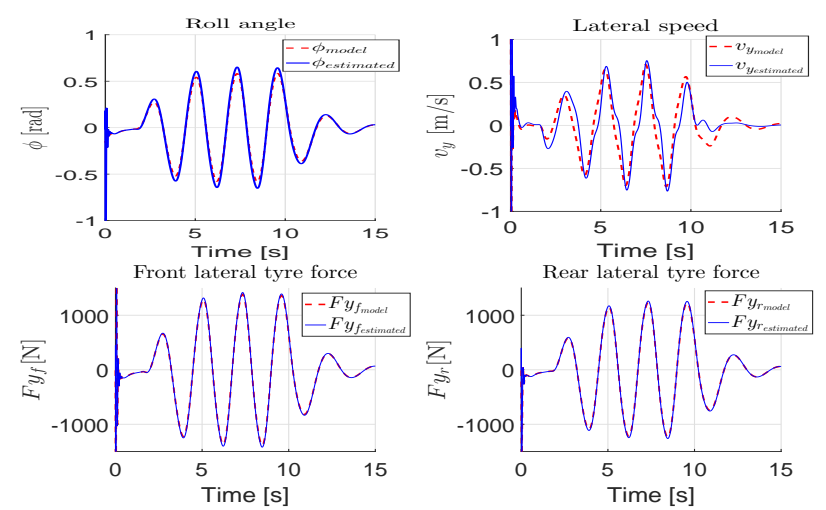

Fig. 5. States estimation compared to model state.

In figures $(3 \sqrt[45]{5}$, we see that the state and variables are well estimated except the lateral velocity which has relative error estimation, but it is still acceptable. The lateral velocity is difficult to estimate accurately. However, because of its low value compared to longitudinal velocity this error does not affect the performances of the state estimation.

\section{B. Motorcycle riding scenario}

This section aims to present experimental results using a scenario realized on urban scenic road and normal riding behavior to validate the proposed observer. The instrumented motorcycle is an Electric Scooter Peugeot model (figure 6) (please refer to [26] for data set and experimentation).
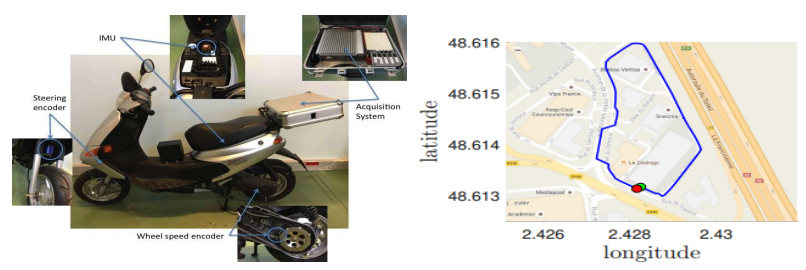

Fig. 6. Scooter and Vehicle trajectory

The observer estimate the lateral dynamics using the measured states $\left(\dot{\phi}, \dot{\psi}, a_{y}, y_{0}=C_{0} x\right)$ given by the inertial unit and $\delta$ obtained from the steering encoder, longitudinal speed obtained from GPS data.

The scenario is considered with a varying longitudinal velocity from $10 \mathrm{~km} / \mathrm{h}$ on $40 \mathrm{~km} / \mathrm{h}$, with the corresponding steering torque depicted in (fig. 7).
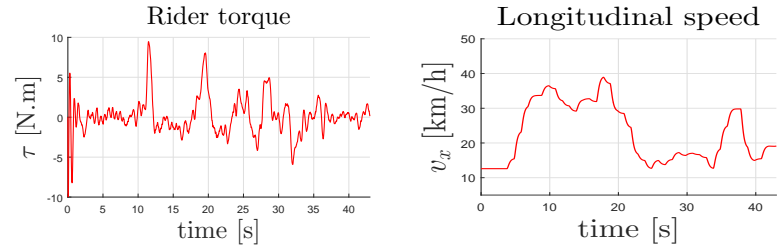

Fig. 7. (left) steering torque (right) longitudinal velocity

The actual signals given by the encoder and the IMU required in the observer design are depicted in (fig. 8 ).
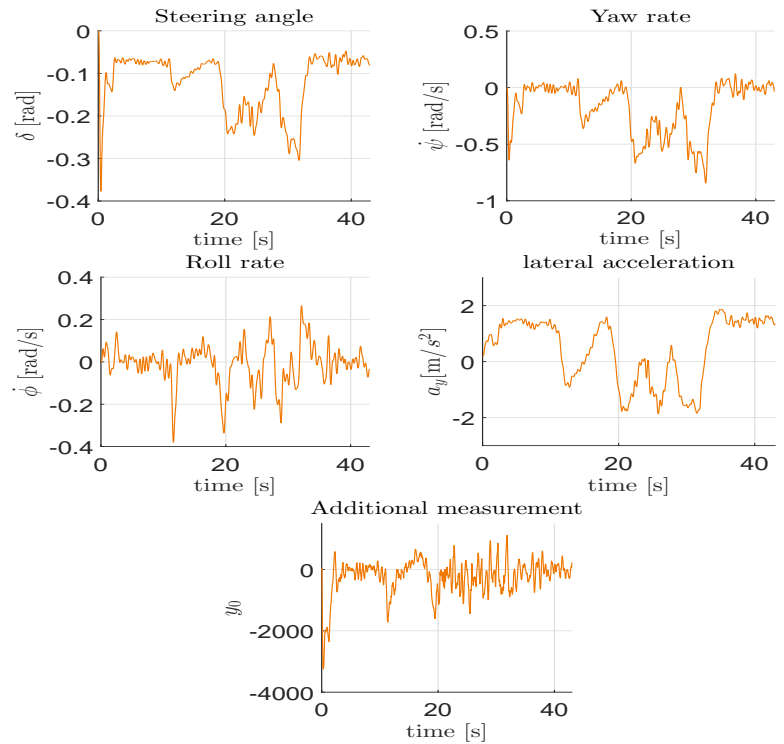

Fig. 8. Measured states 

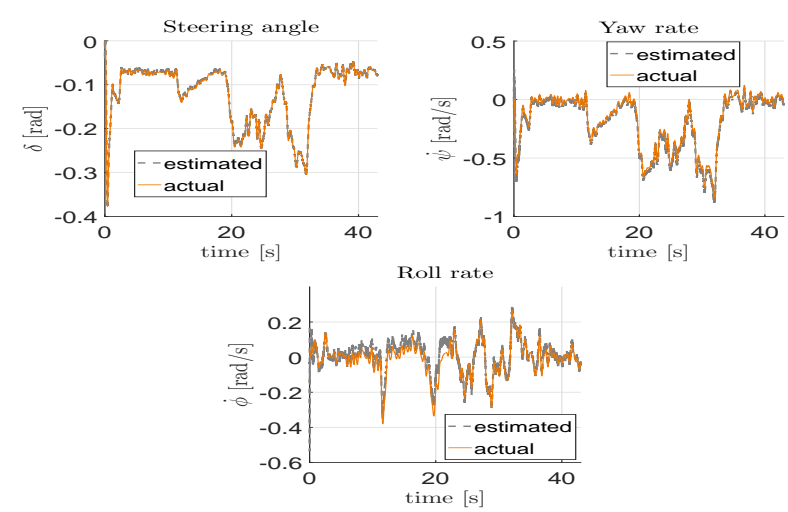

Fig. 9. Validated dynamic states: states estimation (gray) compared to actual measurement (orange).

(fig. 9 and fig. 10) shows the states estimation of the lateral dynamic model, whereas (fig. 12) illustrate the estimation of the tire cornering parameters.
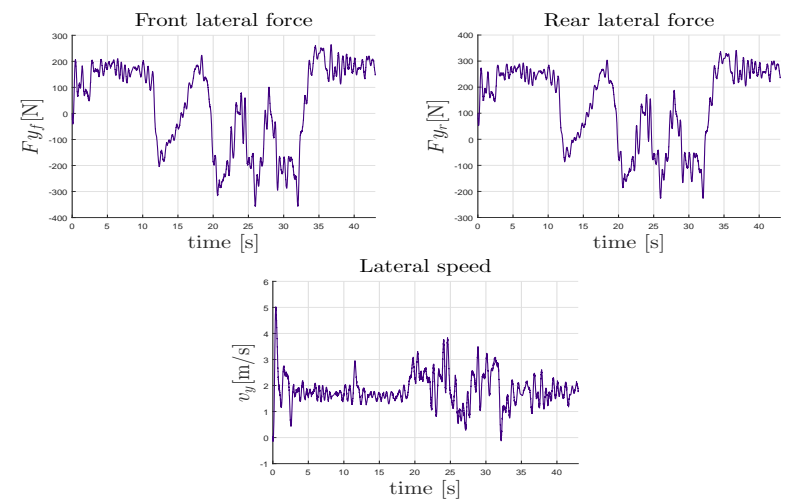

Fig. 10. Unmeasurable states estimation of the scooter.

\section{Validated dynamic states}

Since the actual state $v_{y}$ and $F_{y f}, F_{y r}$ are unknown, the state estimation cannot be checked directly as it was done in figure 9 in the above simulation. To validate the estimation, one can use the estimates state $v_{y}$ and $F_{y f}, F_{y r}$ to construct the lateral acceleration from equations (22) to compare with the measured acceleration as shown in (fig 11). Where, orange: measure $a_{y}$, gray: estimated with sum of the estimated lateral forces and dashed purple: estimated with lateral motion, the additional measure can also be used to verified the lateral forces using equations 8 .

$$
\hat{a}_{y}=\frac{\left(\hat{F}_{y f}+\hat{F}_{y r}\right)}{M}, \hat{a}_{y}=\hat{v}_{y}+v_{x} \dot{\psi}
$$

In (fig 11), the estimate roll angle (gray) is also validated with measured roll angle given by the IMU (orange measure).
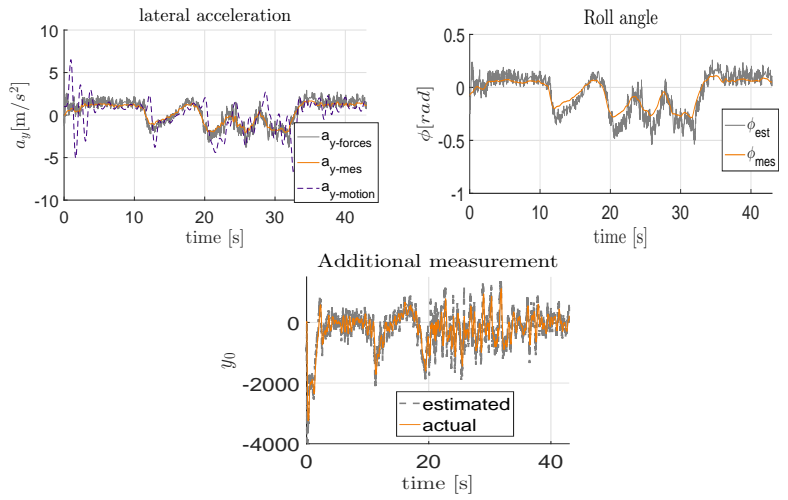

Fig. 11. Validated state estimation $: v_{y}, F_{y f}, F_{y r}$ from the lateral acceleration and the additional measurement; and $\phi$ from IMU roll angle.

These last figures demonstrate the ability of the adaptive observer to estimate the lateral dynamic states on a real riding scenario realized with normal riding behavior.

In figure 12, the tire cornering identification is carried out with motorcycle measurement. Since the ideal parameters in this case are unknown, we evaluate the performance of the adaptive law by comparing the state of the model with the actual output. To validate the identified parameters, one can inserted the identified coefficients into the related models adapted with the estimated coefficient $\left(\hat{C}_{f, r}\right)$, to compare the updated state of the model with the corresponding measurement and checking the similarity (note that this validation is done without estimation), In figure 13, the lateral acceleration computed $M a_{y}=\sum F_{y_{f r}}$ is compared with the actual $a_{y}$, also the roll rate measure compared to roll rate state are shown.
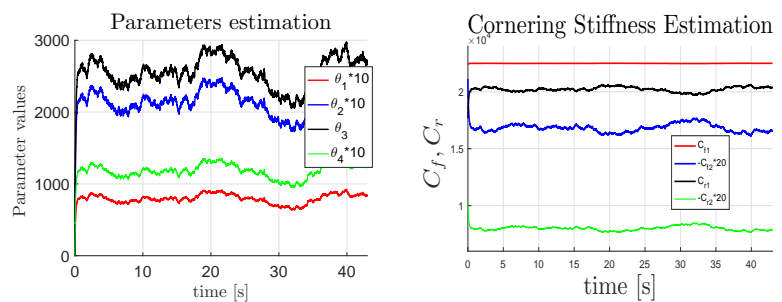

Fig. 12. (left) Convergence of parameters estimates $\hat{\theta}_{1}, \hat{\theta}_{2}, \hat{\theta}_{3}$ and $\hat{\theta}_{4-}$ (right) $\hat{C}_{(f, r) i}=\hat{\theta}_{i}+C_{(f, r) i_{0}}(f, r=$ front, rear $)$ and $i=(1,2,3,4)$
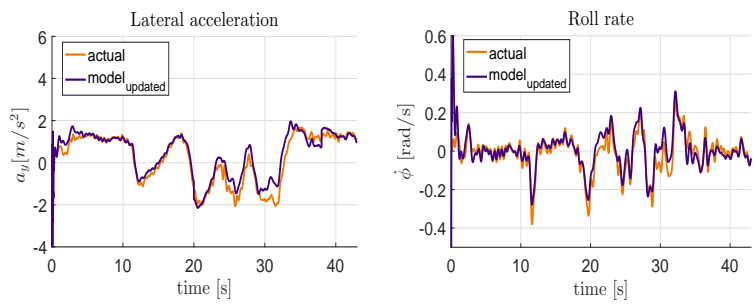

Fig. 13. Validated the tire cornering stiffness with the roll rate measure and lateral acceleration: orange: measure, purple: model updated with $\hat{C}_{f r}$.

These figures show the performance of the used adaptive 
observer to estimate simultaneously the motorcycle dynamics and the identification of tire cornering parameters.

According to the above simulation results, it can be seen that the observer has a good dynamic transition and an acceptable convergence to the estimated value. The simulation results show that the estimated model gives a good representation of the actual states. Note that the lateral velocity is difficult to estimate, however it remains acceptable, the roll and steer angles $(\phi, \delta)$ and also the lateral forces are well estimated, there are some differences at the peak due to modeling errors. The model used for the observer design do not take into account large roll angles but the observer still gives acceptable results.

Remark 2: The major limitation of motorcycle dynamics is due to nonlinear tire characteristics and to loss of friction between tire and road. The lateral friction mainly depends on road condition (dry, wet, snow, ice) and is related to the efforts at the tire level (cornering stiffness). The estimate of the tire coefficient could be used in Advanced Rider Assistance Systems (ARAS) which are based on the detection of loss of tire/road friction in order to provide a warning of a possible loss of adhesion.

The small or negative cornering stiffness means that tire has reached their performance limit (the nonlinear effects like tire saturation), in this case, the ARAS systems derived for motorcycle, can't help the rider to master the critical situations.

The great value of cornering stiffness indicates that the tire characteristics is within the linear range and therefore the equations of the motorcycle model and the control laws obtained with this model are valid.

For this reason it is necessary to incorporate this important tire information into the control law.

\section{CONCLUSION}

In this paper, an estimation of out of plane motorcycle model and tire cornering stiffness coefficient have been proposed using an adaptive observer. The observer has been designed subject to Lyapunov theory and persistence excitation. The design takes into account the forward speed as a linear parameter varying. Sufficient conditions for the existence of the estimator are given in terms of linear matrix inequalities (LMIs). Then, the performance of the resulting observer has been evaluated by simulation using a realistic riding scenario.

Future works will be dedicated to the improvements of the observer performance by adding the rider motion and taking into account road feature. Furthermore a robustness observer analysis including parameters uncertainties on the motorcycle system could be carried out.

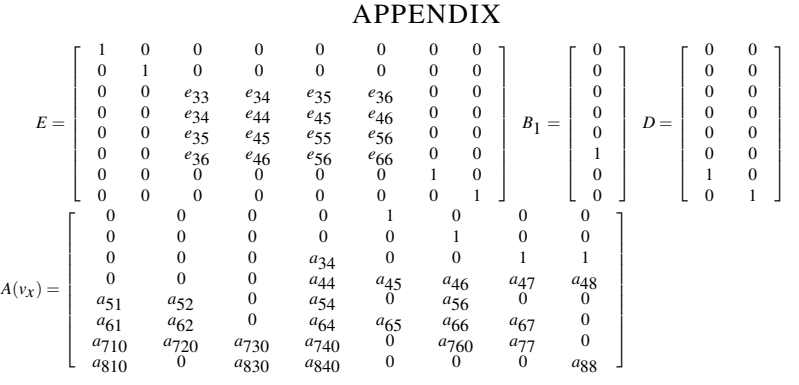

Proof 1: The key step to define the Lipschitz condition for the unknown part of the model $\tilde{f}$ :

1) The linear function $\tilde{f}$ can be thought of as being written in the form

$$
\begin{aligned}
\tilde{f} & =D \chi\left(x, v_{x}\right) \theta-D \chi\left(\hat{x}, v_{x}\right) \theta \\
& =D \tilde{\chi}\left(\tilde{x}, v_{x}\right) \theta
\end{aligned}
$$

2) From equation $7,\left(\tilde{\chi}\left(\tilde{x}, v_{x}\right) \theta\right)$ can be obtained:

$$
\tilde{\chi}\left(\tilde{x}, v_{x}\right) \theta=\left[\begin{array}{c}
-\frac{v_{x}}{\sigma_{f}} \tilde{\alpha}_{f} \theta_{f 1}+\frac{v_{x}}{\sigma_{f}} \tilde{\gamma}_{f} \theta_{f 2} \\
-\frac{v_{x}}{\sigma_{r}} \tilde{\alpha}_{r} \theta_{r 1}+\frac{v_{x}}{\sigma_{r}} \tilde{\gamma}_{r} \theta_{r 2}
\end{array}\right]=\left[\begin{array}{c}
\tilde{\chi}_{1} \\
\tilde{\chi}_{2}
\end{array}\right]
$$

where, the following notation is used :

$$
\left\{\begin{array}{l}
\tilde{x}=\left(\tilde{\phi}, \tilde{\boldsymbol{\delta}}, \tilde{y}_{y}, \tilde{\dot{\psi}}, \tilde{\phi}, \tilde{\dot{\delta}}, \tilde{F}_{y f}, \tilde{F}_{y r}\right) \\
=\left(\tilde{x}_{1}, \tilde{x}_{2}, \tilde{x}_{3}, \tilde{x}_{4}, \tilde{x}_{5}, \tilde{x}_{6}, \tilde{x}_{7}, \tilde{x}_{8}\right) \\
\tilde{\alpha}_{f}=\left(\frac{\tilde{x}_{3}+l_{f} \tilde{x}_{4}-\eta \tilde{x}_{6}}{v_{x}}\right)-\tilde{x}_{2} \cos (\varepsilon) \\
\tilde{\alpha}_{r}=\left(\frac{\tilde{y}_{3}-l_{r} \tilde{x}_{4}}{v_{x}}\right) \\
\tilde{\gamma}_{f}=\tilde{x}_{1}+\tilde{x}_{2} \sin (\varepsilon) \\
\tilde{\gamma}_{r}=\tilde{x}_{1} \\
\tilde{\alpha}_{f}=\left(\alpha_{f}-\hat{\alpha}_{f}\right) \\
\tilde{\alpha}_{r}=\left(\alpha_{r}-\hat{\alpha}_{r}\right) \\
\tilde{\gamma}_{f}=\left(\gamma_{f}-\hat{\gamma}_{f}\right) \\
\tilde{\gamma}_{r}=\left(\gamma_{r}-\hat{\gamma}_{r}\right)
\end{array}\right.
$$

3) If we choose $Q=I_{8}$, one obtain:

$$
\tilde{f}^{T} Q \tilde{f}=\tilde{f}^{T} \tilde{f}=\tilde{\chi}_{1}^{T} \tilde{\chi}_{1}+\tilde{\chi}_{2}^{T} \tilde{\chi}_{2}=\tilde{\chi}_{1}^{2}+\tilde{\chi}_{2}^{2}
$$

4) Then, we can write the following quadratic form :

$$
\tilde{f}^{T} Q \tilde{f} \leq \tilde{x}^{T} R \tilde{x}
$$

Where,

$$
R=\left[\begin{array}{cccccccc}
d_{1} & \frac{d_{12}}{2} & \frac{d_{13}}{d_{2}} & \frac{d_{14}}{d_{12}^{2}} & 0 & \frac{d_{16}}{d^{2}} & 0 & 0 \\
\frac{d_{12}}{2} & \frac{d_{2}}{2} & \frac{d_{23}}{2} & \frac{d_{24}}{2} & 0 & \frac{d_{26}}{2} & 0 & 0 \\
\frac{d_{13}}{2} & \frac{d_{23}}{d_{14}} & d_{3} & \frac{d_{34}}{2} & 0 & \frac{d_{36}}{d_{14}} & 0 & 0 \\
\frac{d_{24}}{2} & \frac{d_{34}}{2} & d_{4} & 0 & \frac{d_{46}}{2} & 0 & 0 \\
0 & 0 & 0 & 0 & 0 & 0 & 0 & 0 \\
\frac{d_{16}}{2} & \frac{d_{26}}{2} & \frac{d_{36}}{2} & \frac{d_{46}}{2} & 0 & d_{6} & 0 & 0 \\
0 & 0 & 0 & 0 & 0 & 0 & 0 & 0 \\
0 & 0 & 0 & 0 & 0 & 0 & 0 & 0
\end{array}\right]
$$

The expressions of $d_{i j}$ are :

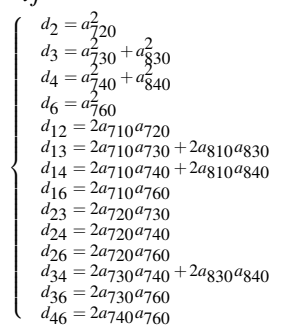




\section{NOMENCLATURE} whole motorcycle

$j, h, k, e, l_{f}, \quad$ linear dimensions

$l_{r}$

$i_{f y}, i_{r y}$

$R_{f}, R_{r}$

K

$I_{f x}, I_{r x}$

$I_{f z}, I_{r z}$

$C_{r x z}$

$Z_{f}$

$\sigma_{f}, \sigma_{r}$
$M_{f}, M_{r}, M \quad$ mass of the front frame, the rear frame and the polar moment of inertia of front and rear wheels radius of front and rear wheels damper coefficient of the steering mechanism front and rear frame inertias about $X$ axis front and rear frame inertias about $Z$ axis rear frame product of inertia w.r.t. $X$ and $Z$ axis front vertical force coefficients of relaxation of the front and rear pneumatic forces

TABLE I

MOTORCYCLE PARAMETERS EXPRESSIONS
[10] Fouka, M., Damon, P. M., Nehaoua, L., Arioui, H., Mammar, S. Parametric Identification of a Powered Two-Wheeled Vehicles: Algebraic Approach. IEEE International Conference: 25th Mediterranean Conference on Control and Automation (MED), 2017.

[11] M. Corno, S.M. Savaresi, and G.J. Balas, On linear parameter varying (LPV) slip controller design for two wheeled vehicles, Int. J. Robust Nonlinear Control, vol.19, no.12, pp. 1313-1336, 2009.

[12] Savaresi, S. M., Tanelli, M., Langthaler, P., Del Re, L. (2008). New regressors for the direct identification of tire deformation in road vehicles via in-tire accelerometers. IEEE Transactions on Control Systems Technology, 16(4), 769-780.

[13] Tanelli, M., Piroddi, L., Savaresi, S. M. (2009). Real-time identification of tireroad friction conditions. IET control theory applications, 3(7), 891-906.

[14] Savaresi, S., Tanelli, M., Langthaler, P., Del Re, L. (2006). Identification of tire-road contact forces by in-tire accelerometers. In 14th IFAC Symposium on System Identification (pp. 1-6).

[15] R. S. Sharp, The stability and control of motorcycles, Mechanical Engineering Science, vol. 13, no. 5, pp. 316 329, 1971.

[16] V. Cossalter, Motorcycle Dynamics. Lulu. com, 2006.

[17] H.B. Pacejka. Tire and Vehicle Dynamics. SAE International, 2005.

[18] Tanaka, K., Wang, H. O. (2004). Fuzzy control systems design and analysis: a linear matrix inequality approach. John Wiley Sons.

[19] Fliess, M., Lvine, J., Martin, P., Rouchon, P. (1995). Flatness and defect of non-linear systems: introductory theory and examples. International journal of control, 61(6), 1327-1361.

$e_{33}=M, e_{35}=M_{f} j+M_{r} h, e_{46}=M_{f} e k+I_{f z} \cos \varepsilon, e_{34}=M_{f} k$,

$e_{44}=M_{f} k^{2}+I_{r z}+I_{f x} \sin ^{2} \varepsilon+I_{f z} \cos ^{2} \varepsilon, a_{44}=-M_{f} k v_{x}, e_{36}=M_{f} e$

$e_{45}=M_{f} j k-C_{r x z}+\left(I_{f z}-I_{f x}\right) \sin \varepsilon \cos \varepsilon, a_{34}=-M v_{x} a_{45}=\frac{i_{f y}}{R_{f}}+\frac{i_{r y}}{R_{r}}$,

$a_{46}=\frac{i_{f y}}{R_{f}} \sin \varepsilon v_{x} e_{55}=M_{f} j^{2}+M_{r} h^{2}+I_{r x}+I_{f x} \cos ^{2} \varepsilon+I_{f z} \sin ^{2} \varepsilon$,

$e_{56}=M_{f} e j+I_{f z} \sin \varepsilon, a_{47}=l_{f}, a_{48}=-l_{r}, a_{51}=\left(M_{f} j+M_{r} h\right) g$,

$a_{54}=-\left(M_{f} j+M_{r} h+\frac{i_{f y}}{R_{f}}+\frac{i_{r y}}{R_{r}}\right) v_{x}, a_{56}=-\frac{i_{f y}}{R_{f}} \cos \varepsilon v_{x}$,

$a_{52}=M_{f} e g-\eta Z_{f}, a_{61}=M_{f} e g-\eta Z_{f}, a_{62}=\left(M_{f} e g-\eta Z_{f}\right) \sin (\varepsilon)$

$e_{66}=I_{f z}+M_{f} e^{2}, a_{64}=-\left(M_{f} e+\frac{i_{f y}}{R_{f}} \sin \varepsilon\right) v_{x}, a_{65}=\frac{i_{f y}}{R_{f}} \cos (\varepsilon) v_{x}$,

$a_{66}=-K, a_{67}=-\eta, a_{710}=\frac{C_{f 20}}{\sigma_{f}} v_{x}, a_{730}=-\frac{C_{f 10}}{\sigma_{f}}, a_{740}=-\frac{C_{f 10}}{\sigma_{f}} l_{f}$,

$a_{720}=\frac{\left(C_{f 10} \cos (\varepsilon)+C_{f 20} \sin (\varepsilon)\right)}{\sigma_{f}} v_{x}, a_{760}=\frac{C_{f 10}}{\sigma_{f}} \eta ; a_{770}=-\frac{1}{\sigma_{f}} v_{x}, a_{810}=\frac{C_{r 20}}{\sigma_{r}} v_{x}$, $a_{83}=-\frac{C_{r 10}}{\sigma_{r}}, a_{840}=\frac{C_{r 10}}{\sigma_{r}} l_{r}, a_{880}=-\frac{1}{\sigma_{r}} v_{x}$,

\section{REFERENCES}

[1] B. Evangelos, Saferider project, French National Agency of Reaserch, Tech. Rep., 2010.

[2] N. Haworth, P. Rowden, B. Watson, D. Wishart, L. Buckley, and K. Greig, Motorcycle rider safety project, The Centre for Accident Research and Road Safety Queensland, Tech. Rep., 2012.

[3] L. Gasbarro, A. Beghi, R. Frezza, F. Nori, and C. Spagnol, Motorcycle trajectory reconstruction by integration of vision and MEMS accelerometers, in Conference on Decision and Control, 2004.

[4] A. P. Teerhuis and S. T. H. Jansen, Motorcycle state estimation for lateral dynamics, Vehicle System Dynamics, vol. 50, no. 8, pp. 12611276, 2012.

[5] D. Ichalal, C. Chabane, H. Arioui, and S. Mammar, Estimation de la dynamique laterale pour vehicules 'a deux roues motorises, in Septi'eme Conference Internationale Francophone dAutomatique, 2012.

[6] M. E. H. Dabladji, D. Ichalal, H. Arioui, and S. Mammar, Observer based controller for single track vehicles, in proc. of the IEEE Conference on Decision and Control, 2013.

[7] P. De Filippi, M. Corno, M. Tanelli, and S. Savaresi, Single-sensor control strategies for semi-active steering damper control in twowheeled vehicles, Vehicular Technology, IEEE Transactions on, vol. 61, no. 2, pp. 813820, 2011.

[8] L. Nehaoua, D. Ichalal, H. Arioui, J. Davila, S. Mammar, and L. Fridman, An unknown input HOSM approach to estimate lean and steering motorcycle dynamics, Vehicular Technology, IEEE Transactions on, 2013.

[9] Fouka, M., Nehaoua, L., Arioui, H., Mammar, S. "Mutiple-Gradient Descent Algorithm for Parametric Identification of a Powered TwoWheeled Vehicles". IEEE International Conference on Systems, Man, and Cybernetics (SMC), 2017.
[20] Levant, A. (2003). Higher-order sliding modes, differentiation and output-feedback control. International journal of Control, 76(9-10), 924-941.

[21] Pertew, A. M., Marquez, H. J., Zhao, Q. (2005, June). Design of unknown input observers for Lipschitz nonlinear systems. In American

1 Control Conference, 2005. Proceedings of the 2005 (pp. 4198-4203). IEEE.

[22] Xie, L."Output feedback $H_{\infty}$ control of systems with parameter uncertainty". International Journal of Control 1996.

[23] A. Ioannou, Jing Sun." Robust Adaptive Control". Courier Dover publications, 2012.

[24] Sharp, R. S., Evangelou, S., Limebeer, D. J. (2004). Advances in the modelling of motorcycle dynamics. Multibody system dynamics, 12(3), 251-283.

[25] Corless, M., Tu, J. A. Y. (1998). State and input estimation for a class of uncertain systems. Automatica, 34(6), 757-764.

[26] Damon, P. M., Ichalal, D., Nehaoua, L., Arioui, H. (2017). Lateral Steering Dynamics Estimation for Single Track Vehicle: Experimental Tests. IFAC-PapersOnLine, 50(1), 3400-3405. 